\title{
Das verschmähte Instrument der (Fernstraßen- oder City-) Maut
}

\section{Gernot Sieg}

Online publiziert: 29. September 2020

(C) Der/die Autor(en) 2020

Zusammenfassung Seit langem werden von Ökonomen Straßenbenutzungsgebühren als Instrument gegen Verkehrsstaus empfohlen. In der Praxis gibt es jedoch nur wenige Anwendungen. In diesem Artikel werden die unterschiedliche Stautechnologien und die jeweils adäquate Maut identifiziert. Widerstände, die einer Maut entgegenstehen, und Möglichkeiten, sie zu überwinden, werden aufgezeigt. Es ist an der Zeit, den Einheitlichen Europäischen Mautdienst zu realisieren und einen Rechtsrahmen in Deutschland zu schaffen, der es Städten erlaubt, Maut zu erheben.

Schlüsselwörter Verkehrsstau $\cdot$ Maut $\cdot$ Hyperstau $\cdot$ EEMD

\section{Congestion charges: a despised instrument}

Abstract Road tolls have long been recommended by economists as a remedy against traffic congestion. In practice, however, there are few applications. In this article, different congestion technologies and appropriate tolls are identified. Resistances that stand in the way of a toll and ways of overcoming them are shown. It is time to implement the European Electronic Toll Service and create a legal framework in Germany that allows cities to collect tolls.

\section{Einleitung}

Verkehrsstaus sind die ständigen Begleiter von Teilnehmern des motorisierten Individualverkehrs. Für viele Ökonomen ist der Grund die fehlende oder falsche Bepreisung der Straßennutzung. So identifiziert Vickrey $(1963,452)$, ,[...] in no other 
major area are pricing practices so irrational, so out of date, and so conducive to waste as in urban transportation“" und schlägt eine Internalisierung der Überlastungsexternalitäten im Stile einer Pigou-Steuer vor. In dieselbe Kerbe schlagen die wissenschaftlichen Beiräte beim BMVI und beim BMWi, der Deutsche Städtetag, die EU Kommission, Cramton et al. (2018) und ganz aktuell ein gemeinsames Plädoyer für City-Maut initiiert von RWI Leibniz-Institut für Wirtschaftsforschung und der Stiftung Mercator.

In der Praxis finden sich jedoch nur vereinzelt Anwendungen: So gibt es in Deutschland eine der EU-Wegekostenrichtlinie (2006/38/EG) entsprechende Maut für Lkw deren zulässiges Gesamtgewicht mindestens 7,5 Tonnen beträgt auf Bundesfernstraßen, die die Kosten für die Infrastruktur und die externen Kosten von Lärm und Luftschadstoffen umlegt (Bundesfernstraßenmautgesetz - BFStrMG). Eine belastungsabhängige Differenzierung nach der Tageszeit, der Tageskategorie oder der Jahreszeit, die die Richtlinie unter Bedingungen ${ }^{1}$ ermöglicht, findet in Deutschland jedoch nicht statt. Ein Rechtsrahmen für die Erhebung einer City-Maut wäre notwendig (Wissenschaftlicher Dienst des Bundestages 2013), existiert jedoch nicht. In den USA gibt es High Occupancy Toll Lanes, d.h. gebührenpflichtige Fahrstreifen die parallel zu gebührenfreien Fahrstreifen verlaufen. ${ }^{2}$ Singapore besitzt ein „Electronic Road Pricing“, bei dem die Gebühren auf Highways abhängig sind von Pkw-Typ, der Tageszeit und dem Ort. Citymauten für Fahrten innerhalb eines Gebiets (Area Pricing) gibt es in London, Durham, Bologna und Mailand mit Tagespreisen und in Valletta (zeitabhängig innerhalb der Zone), während des Überfahren eines Rings (Cordon Pricing) in Stockholm und Göteborg (jeweils tageszeitabhängig) bepreist wird. New York plant eine 12 Dollar Gebühr für Fahrten in Manhattan südlich der 61. Straße mit Ausnahmen ${ }^{3}$.

Die Straßentechnologie unterscheidet sich in Städten und auf der Autobahn, und so unterscheiden sich auch die Ursachen von Staus (Vickrey 1969, 2019). Die Überlastungsexternalität tritt schon auf, wenn ein einzelnes langsam fahrendes Kraftfahrzeug einen anderen Pkw dazu veranlasst, langsamer zu fahren als gewünscht und so mehr Fahrzeit aufzuwenden als bei Abwesenheit dieses Kraftfahrzeugs. Viele Fahrzeuge beeinflussen sich gegenseitig, so dass die erreichbare Geschwindigkeit mit einem höheren Verkehrsfluss (Kfz/Stunde) sinkt. Bei einem bottleneck oder Verkehrsengpass (der Elbtunnel an Ferienwochenenden, eine angekündigte Tagesbaustelle oder die Fahrstreifensperrung durch einen Unfall) ist die Anzahl der Kfz, die den Engpass passieren wollen, größer als die Engpasskapazität und vor dem Engpass entsteht eine Warteschlange. Diese Warteschlange kann so lang werden, dass selbst

\footnotetext{
1 (1) Keine Mautgebühr liegt mehr als 100 Prozent über der Gebühr, die während der billigsten Tageszeit, Tageskategorie oder Jahreszeit erhoben wird. (2) Wenn für den billigsten Zeitraum der Nulltarif gilt, darf der Zuschlag für die teuerste Tageszeit, Tageskategorie oder Jahreszeit 50 der normalerweise für das betreffende Fahrzeug zu entrichtenden Mautgebühr nicht überschreiten.

2 Casady et al. (2020) untersuchen Nutzen und Kosten von 7 HOT-Lanes in den USA und identifizieren nur für zwei einen Nutzen, der über den Kosten liegt. Die Ergebnisse sind abhängig von den einbezogenen Nutzen- und Kostenkomponenten, beispielsweise fehlt eine etwaige Steigerung der Zuverlässigkeit, und die Bewertung der Komponenten. Dennoch zeigt die Untersuchung, dass nicht jede Umsetzung der Maut eine potentielle Pareto-Verbesserung bedeutet.
}

3 FDR Drive and West Side Highway, Anwohner und Personen mit Einkommen unter 60.000 Dollar. 

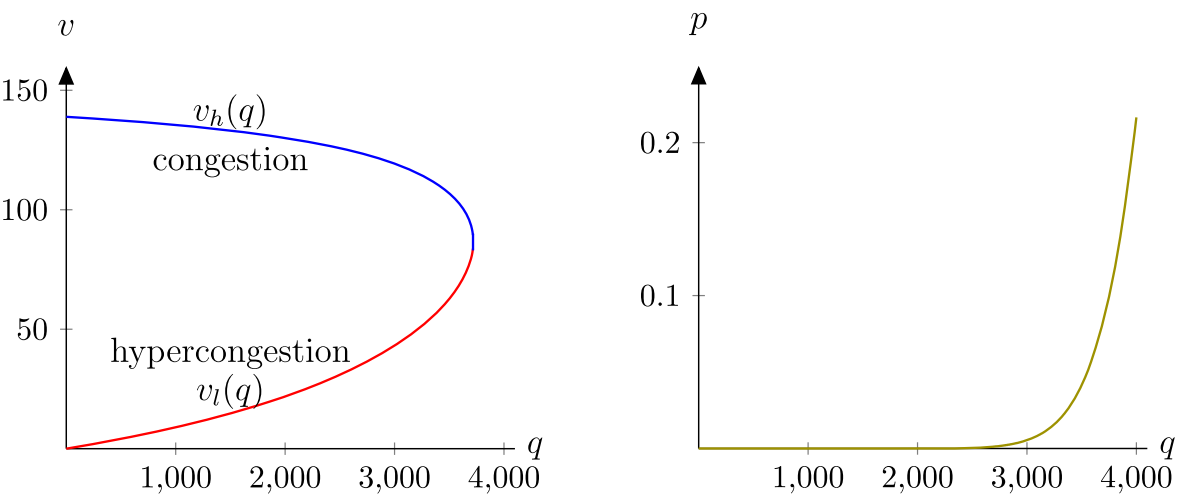

Abb. 1 Fundamentaldiagramm: Geschwindigkeit $v$ ist abhängig vom Verkehrsfluss $q$

die Kfz, die den Engpass (Elbtunnel in Richtung Norden) nicht passieren sondern vorher (Ausfahrt HH-Waltershof) abbiegen wollen, warten müssen (Triggerneck). Im stop-and-go Verkehr ist die Kapazität der Straße nur noch ineffizient gering so dass man von Hyperstau redet. Auch entstehen Staus ohne ersichtlichen Grund, nur weil die interagierenden Fahrzeuge sich durch Bremsen, Beschleunigen, langsame Überholvorgänge von Lkw oder Fahrspurwechseln so beeinflussen, dass ein sogenannter Phantom-Stau entsteht (Sugiyama et al. 2008; Nakayama et al. 2009; Tadaki et al. 2013). In überlasteten Städten passen die Fahrer ständig die Routen an die Stausituation an, so dass sich der Stau gleichmäßig (und nicht hinter einem bottleneck) ausbreitet. Nicht nur auf Autobahnen sondern auch auf (aggregiertem) Stadtniveau gilt das Fundamentaldiagramm (siehe Abb. 1), d.h. eine stabile Beziehung zwischen Verkehrsfluss in der Stadt und durchschnittlicher Geschwindigkeit (Daganzo 2007; Geroliminis und Daganzo 2008; Daganzo et al. 2011). Darüber hinaus existieren Netzwerk- oder Kontrollstaus, wenn Regelungen für die Hauptverkehrszeit (Tempolimit, Lichtzeichenanlagen) auch während der Nebenzeit gelten, dort aber eigentlich überflüssig sind. Eine intermodale Externalität entsteht, wenn ein Kfz beispielsweise vor Bahnschranken halten müssen, da eine Eisenbahn passiert.

Damit eine Maut Stau reduziert, muss sie das Verhalten der Konsumenten ändern:

1. Die Konsumenten können ihre Route ändern, und anstelle von hochbelasteten Straßen weniger belastete Ausweichstrecken wählen (reassignment). Selbst ohne Maut werden schon heute von vielen Verkehrsteilnehmern Navigationsapps genutzt, die Daten in Echtzeit verarbeiten und zum Zwecke der Fahrtzeiteinsparung Alternativstrecken empfehlen. Oft reichen wenige Ausweichstreckennutzer aus, um auf allen möglichen Routen fast gleichlange Fahrzeiten zu erreichen. Ein weiteres Optimierungspotential durch eine Maut besteht dann jedoch nicht mehr.

2. Die Konsumenten könnten ihr Ziel ändern (redistribution). Insbesondere beim Zweck Freizeit, Urlaub oder Einkaufen wäre diese Verhaltensänderung denkbar. Gerade die Zielgebiete wie Urlaubsregionen oder Einkaufsstädte fürchten deshalb, unter einer solchen Maut zu leiden, wenn sie nur regional eingeführt wird. Eine überregionale Koordination könnte unerwünschte Zieländerungen jedoch verhin- 
dern. Um Pendler dazu zu bewegen, den Arbeits- oder Wohnort zu ändern, sind im Vergleich größere Anreize notwendig und der Wechsel benötigt mehr Zeit.

3. Die Konsumenten könnten ihre Abfahrtszeit ändern (rescheduling). Ein Arbeitnehmer mit flexibler Arbeitszeit beispielsweise könnte früher als bevorzugt zur Arbeit fahren, um den Stau zu vermeiden, während ein Arbeitnehmer mit festen Arbeitszeiten gezwungen ist, pünktlich anzukommen. Auch könnte man die Abfahrtszeiten der Engpasskapazität anpassen und so Staus verhindern.

4. Die Konsumenten könnte ihren Verkehrsmodus ändern (mode-switching), und vom Pkw aufs Fahrrad, das Motorrad oder den ÖPNV wechseln oder sogar zu Fuß gehen. Entscheidend für den Wechsel zum ÖPNV sind neben dem Preis die Fahr- und Wartezeiten, die Frequenz, die Sicherheit und andere Komfortparameter und somit die Qualität des ÖPNV Angebots. Beim Wechsel zum Fahrrad spielt die Entfernung, Geographie, das Wetter und die Fahrradinfrastruktur als Bestimmungsgrund der Fahrradsicherheit eine Rolle. In der Regel ist der Pkw schon ohne Maut die teurere Alternative, so dass im Status quo neben dem Preis die oben genannten anderen Gründe entscheidungsrelevant sind. Eine alleinige Veränderung der Höhe des Preises für die Pkw-Nutzung durch eine Maut ist deshalb zuerst bei einkommensschwachen Gruppen erfolgreich.

5. Die Konsumenten könnten den Besetzungsgrad ändern (car-pooling). Durch das gemeinsame Nutzen eines Pkw bei ähnlichen Streckenbedürfnissen können die Mautkosten aufgeteilt werden. Unabhängig von einer Maut werden die Pro-KopfBetriebskosten durch car-pooling stark vermindert, während durch Wartezeiten und Umwege aufgrund unterschiedlicher Start- oder Zielpunkte die Fahrzeitkosten ansteigen. Auf das Car-sharing hätte eine streckenabhängige Maut keinen Einfluss, bei einer Zonentagesmaut, oder einer Cordonmaut könnten das Car-sharing durch eine Mautaufteilung sogar die Verkehrsleistung erhöhen, weil in der Realität das Fahrzeug (und nicht ggf. wechselnden Fahrer) mautpflichtig sind.

6. Die Konsumenten könnten die Frequenz der Fahrten ändern, in dem sie durch home office das Pendeln vermeiden, oder mehrere Einkaufsfahrten bündeln.

7. Die Konsumenten könnten gar nicht mehr fahren (trip suppression). Nur solange die Mobilität unverändert bleibt, ist diese Reaktion unbedenklich. Der Effekt ist jedoch kritisch zu sehen, wenn bestimmten Gruppen die soziale Teilhabe verwehrt wird oder die positiven Effekte der Mobilität nicht mehr realisiert werden.

Zusammengefasst sind Mode-switching auf Fahrrad bzw. ÖPNV und rescheduling die gewünschten und am ehesten zu erwarteten Reaktionen. Bei der Antizipation von Mautreaktionen sollte dieses beachtet, denn eine vereinfachte Analyse über das „Gesetz der Nachfrage“, d. h. dass ein höherer Preis (Maut) die Nachfrage reduziert, wird der Komplexität der Verkehrsmärkte nicht gerecht und kann zu nicht adäquaten Politikempfehlungen führen. 


\section{2 Ökonomische Theorie der Verkehrsüberlastung}

\subsection{Internalisierung der Überlastungsexternalität durch eine Pigou-Steuer}

Die Geschwindigkeit $v$ sinkt im Falle der Überlastung (congestion) mit dem Verkehrvolumen $q$ gemessen in Pkw pro Stunden (siehe Fundamentaldiagramm in Abb. 1). Da die Fahrtkosten abhängig von der Fahrzeit sind, und die Fahrzeit mit sinkender Geschwindigkeit zunimmt, erhält man Kosten $c(q)$ abhängig vom Verkehrsvolumen. Die Kosten sind sowohl die privaten Kosten eines Konsumenten wie auch die Durchschnittskosten aller Konsumenten $q$, die die Straße nutzen. Die gesamten soziale Kosten ergeben sich zu $C=c(q) \cdot q$, so dass die sozialen Grenzkosten

$$
\frac{d C}{d q}=c(q)+q \cdot \frac{d c}{d q}
$$

betragen. Da $c(q)$ die privaten Kosten des Konsumenten sind, werden sie bei der Fahrentscheidung internalisiert. $q \cdot d c / d q$ werden jedoch nicht internalisiert, sind also externe Grenzkosten, die mit einer Belastungsmaut internalisiert werden könnten. Die externen Grenzkosten entstehen, weil ein weiteres Fahrzeug $\Delta q$ die Geschwindigkeit aller Fahrzeuge reduziert und mit der gesunkenen Geschwindigkeit steigen die Fahrtkosten für ein Kfz $\Delta c$ an. Da alle Verkehrsteilnehmer betroffen sind, muss man den Effekt mit der Anzahl der Kfz $q$ multiplizieren.

Leider bereitet der Bereich der hypercongestion ein Problem: Die Kostenkurve ist im Bereich der hypercongestion rückwärts geneigt, eine höherer Verkehrsfluss reduziert die Kosten, was der Logik widerspricht. Man könnte hypercongestion als ineffizient und dynamisch instabil (Verhoef 2005) ignorieren, dennoch ist hypercongestion empirisch zu beobachten.

Seit Sugiyama et al. (2008); Nakayama et al. (2009) and Tadaki et al. (2013) ist das Phänomen des Phantom-Staus bekannt. Brilon et al. (2005) und Brilon et al. (2007) und Brilon und Geistefeldt (2010) interpretieren die Kapazität einer Straße als stochastisch, weil die Verkehrsflusszusammenbrüche, die Phantom-Staus, zwar deterministische Gründe wie individuelle Fehlentscheidungen, zu starkes Bremsen oder Überholen von Lkw besitzen kann, aus Systemsicht aber nicht vorhersehbar ist. Goldmann und Sieg (2020a) modellieren das System Straße als ein stochastisches. Die Kapazität einer Straße ist nicht die größtmögliche Verkehrsstärke auf dieser Straße, sondern eine Verkehrsstärke, die mit einer bestimmten Zusammenbruchswahrscheinlichkeit auf einem Streckenabschnitt erreicht werden kann. Abhängig vom Verkehrsfluss ist mit Zusammenbruchswahrscheinlichkeit $p$ der Verkehr zusammengebrochen (hypercongestion) und mit der Wahrscheinlichkeit $1-p$ nicht (congestion). Angewendet auf ein Teilstück der A42 kann man eine Kostenfunktion berechnen, die nicht rückwärts geneigt ist (Goldmann und Sieg 2020b). Die durch eine Maut zu internalisierenden externen Staukosten ergeben sich, je nach gleichgewichtigem Verkehrsfluss, im Bereich von 20 Cent pro km. Der Abfluss aus dem Hyperstauzustand ist jedoch kleiner als der freie Zufluss, so dass die Kapazität sinkt (capacity-drop) (Hall und Agyemang-Duah 1991; Treiber und Kesting 2013; Yuan 
Abb. 2 Grenz- und Durchschnittskosten A42. Quelle: Goldmann und Sieg (2020b)

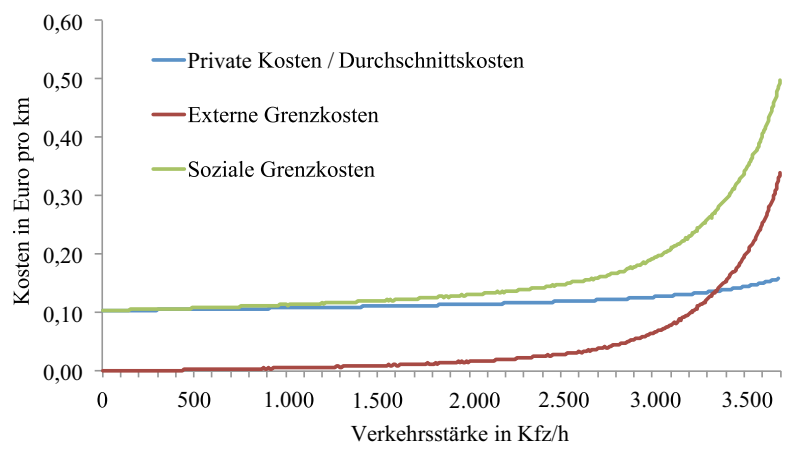

et al. 2017). Ein capacity-drop von 10 Prozent kann dabei (Goldmann und Sieg 2020b) die Stauexternalität auf über 30 Eurocent pro km erhöhen (siehe Abb. 2).

Der Vorteil der Internalisierung ist eine bessere Nutzung der Kapazität Straße, so dass die Gesamtwohlfahrt steigt. Die Gewinner sind die Pendler mit hohen Zeitopportunitätskosten. Dennoch bestehen folgende Befürchtungen (Evans 1992): 1. Eine Pareto-Verbesserung wäre möglich, dazu müssten aber die Mauteinnahmen entsprechend verteilt werden. Die Erfahrungen der Lkw-Maut in Deutschland lassen jedoch daran zweifeln. Die Investitionen des Bundes in Bundesfernstraßen blieben, trotz Einführung der Lkw-Maut mit von knapp 3 Mrd. Euro in 2005 ausgehend steigenden Einnahmen bis 2015 weitgehend ${ }^{4}$ konstant, denn parallel zur Mauteinnahmenerzielung wurden im großen Umfang Steuermittel aus dem Etat abgezogen (Puls 2015). Von daher kann man befürchten, dass die Maut eine Umverteilung zu Lasten der Verkehrsteilnehmer nach sich zieht. 2. Je nach Elastizität der Nachfragefunktion kann der Wohlfahrtsgewinn klein sein, während die Mauteinnahmen und damit der Umverteilungsspielraum sehr groß ist. Wohlfahrtsverluste bei der Verteilung oder Verausgabung der Mauteinnahmen verringern den Gesamteffekt. 3. Die Mauteinnahmen sind höher, wenn Kapazität ineffizient klein ist. Dieses führt, für einen einnahmenorientierten Entscheider, zu perversen Anreizen für Investitionen.

\subsection{Bottleneck}

Ist die Engpasskapazität kleiner als die Nachfrage, so entsteht vor dem Engpass eine Warteschlange (Vickrey 1969). Wenn die Engpasskapazität konstant ist, benötigen die Nutzer eine gewisse Zeit, um den Engpass zu passieren. Diese Zeit lässt sich nicht ändern. Jedoch lässt sich die Wartezeit vor dem Engpass ändern. Kämen alle Nutzer nur in solchen Mengen an, die der Kapazität entspricht, würde keine Warteschlange entstehen und die Wartekosten wären kleiner. Ein Reservierungssystem beispielsweise in Restaurants kann so Warteschlangen komplett eliminieren ohne dass die Anzahl der bedienten Kunden oder Zeiten, an denen gegessen wird, verändert wird.

\footnotetext{
4 Ausnahme waren die Konjunkturpakete I und II.
} 
Arnott et al. (1993) bestimmen eine zeitabhängige Maut, die dazu führt, dass sich die Abfahrtszeiten der Engpassnutzer so ändern, dass die Warteschlange vollständig verschwindet. Die Wartekosten werden in Mauteinnahmen umgewandelt, so dass sich niemand (der homogenen Konsumenten) schlechter stellt, selbst wenn die Mauteinnahmen nicht zurückerstattet werden. Das Modell ist geeignet für Engpässe und Pendeln in die Stadt. Für andere Verkehrszwecke jedoch kommt das Modell an seine Grenzen. Für Güterverkehr besteht oft nicht die Möglichkeit, die Ankunftszeiten am Engpass zu ändern, denn Arbeitszeitbeginn und Arbeitszeitende bestimmen die Routenplanung und die Ankunftszeiten an in der Regel mehreren zu passierenden Engpässen können nur innerhalb eines sehr begrenzten Zeitrahmens geändert werden. Ähnliches gilt für den gewerblichen Verkehr. Eine Dienstwagenbereitstellung durch den Arbeitgeber auch für private Zwecke (Pendeln zur Arbeit) mit der häufig praktizierten kompletten Übernahme aller Betriebskosten durch den Arbeitgeber bei pauschaler Berechnung des damit verbundenen geldwerten Vorteils führt dazu, dass Mautzahlungen für den Arbeitnehmer, der die Routen und Abfahrtzeiten wählt, keine Grenzkosten darstellen. Weil der Arbeitgeber etwaige Mautzahlungen übernimmt, ändert die Maut nicht das Verhalten der Dienstwagennutzer.

Es ist zu erwarten, dass die Maut indirekt regressiv wirkt (Goldmann und Sieg 2021). Ohne Maut erhält man die guten Ankunftszeiten durch längeres warten. Dazu sind die Personen mit relativ hohen Kosten des zu früh oder zu spät Kommens und deshalb relativ geringeren Wartezeitkosten bereit. Mit der Maut werden die guten Zeiten von denen genutzt, die die absolut größeren Kosten des zu früh oder zu spät Kommens besitzen. Das könnte bedeuten, dass die die einkommensschwachen ohne Maut aufgrund ihrer relativ geringeren Wartekosten zu den guten Zeiten ankommen, während sie durch die Maut von den guten Zeiten verdrängt werden. Bei Heterogenität der Nutzer kann eine Maut ihre Eigenschaft der Pareto-Verbesserung verlieren und dazu führen, dass die einkommensschwachen Wohlfahrtsverluste erleiden.

Als Ausweg wird oft vorgeschlagen, die Mauteinnahmen zum Ausbau und zur Subventionierung des ÖPNV zu verwenden. Da das ÖPNV Netz jedoch nicht so engmaschig ist, dass alle Pendler erreicht werden, kann es immer auch einkommensschwache Pendler geben, die weiter mit dem Pkw fahren müssen. Sie fahren wegen der Maut auch nicht mehr zu einer guten Zeit, sondern müssen sehr früh oder sehr spät fahren. Goldmann und Sieg (2021) berechnen in einem Beispiel mit drei unterschiedlichen gleich großen Nutzergruppen, dass die einkommensschwachen Nutzer nur dann von der Maut profitieren, wenn mehr als 15 Prozent aller (oder mehr als 43 Prozent der einkommensschwachen) zum ÖPNV wechseln. Man sollte also versuchen, mit den Mauteinnahmen direkte Anreize zu schaffen, dass engpassnutzende Fahrer auf den ÖPNV wechseln und darauf achten, dass die Mitnahmeeffekte bei der ÖPNV Subventionierung möglichst klein ausfallen.

\subsection{Bathtub}

Geroliminis und Daganzo (2008) identifizieren ein makroskopisches Fundamentaldiagram für die Stadt Yokohama (Japan) und bereiten so einen neuen Weg, wie Überlastung in der Stadt analysiert werden kann. Der Stau im Stadtviertel breitet sich, wie in einer Badewanne der Wasserspiegel, gleichmäßig aus und ist unabhän- 
gig von speziellen Engpässen und Straßen. Die Anpassungsfähigkeit der Autofahrer ist so groß, dass auf allen Verbindungen von Start und Ziel (im Stau) die selbe Fahrzeit erzielt werden kann, und da dass von allen über die ganze Stadtviertel verbreiteten Start und Zielpunkten gilt, ist auch die Überlastung im ganzen Stadtviertel gleich hoch. Das makroskopische Fundamentaldiagram zeigt nun, dass es ab einer bestimmten Nachfrage zu einem ineffizienten Rückgang des Outputs (beendete Fahrten) kommt. Eine Nachfragesteuerung mit Ampeln (die Nachfrage in Warteschlangen umwandeln) oder Zugangsreduktion durch eine Maut ist sinnvoll (Arnott 2013; Fosgerau und Small 2013; Fosgerau 2015; Arnott et al. 2016). So wird hypercongestion verhindert.

Anwendung findet diese Idee beim Bemauten eines gewissen Bereichs (Area Pricing) wie mit Tagespässen in London, Durham, Bologna und Mailand und zeitabhängig innerhalb von Valetta oder aber das bemauten von Überfahrt einer Grenze (Cordon Pricing) wie in Stockholm und Göteborg (jeweils mit tageszeitabhängiger Höhe). Wesentlicher Unterschied ist, dass das Cordon Pricing den Ein- und Auspendlerverkehr verändert, während das Area-Pricing auch den Verkehr innerhalb der Zone bepreist. Das Auseinanderfallen von Betroffenen (Einpendlern) und Entscheidungsberechtigten (Stadtbewohnern) kann zu Ineffizienzen (sog. Ausländermaut) führen. Auch stehen Städte im Wettbewerb um Besucher (Freizeit, Einkaufen) und nutzen schon heute beispielsweise Parkkosten als Wettbewerbsparameter. Ein abgestimmtes Verhalten der Städte bei der Einführung einer Maut kann negative Wettbewerbseffekte verringern. Beide Aspekte sprechen dafür, allgemeine Regeln zur Maut auf übergeordneter Ebene (Bundesland, Bund, Europa) einzuführen.

Die in der Theorie zu erwarteten Ergebnisse zeigen sich auch in Göteborg (West und Börjesson 2020). Der Wohlfahrtsgewinn pro Jahr beträgt 20 Millionen Euro. Die Einrichtungskosten konnten durch die Einnahmen des ersten Jahres beglichen werden, die Wohlfahrt wird nach 4 Jahren positiv. Die Maut ist indirekt regressiv. Die niedrigen Einkommensklassen sind stark abhängig vom Pkw, da Göteborg relativ dünn besiedelt ist und damit das Angebot an ÖPNV eher niedrig ist. Höhere Einkommensgruppen haben oft Zugang zu Dienstwagen und sind deswegen nicht von der Maut betroffen, da die Firma die Maut (für alle Zwecke wie auch Pendeln zur Arbeit oder Freizeit oder Einkauf) übernimmt. Darüber hinaus werden für den Wohlfahrtsgewinn von 20 Millionen Euro (pro Jahr) Mauteinnahmen von fast 77 Millionen Euro (pro Jahr) generiert. Das Überlastungsproblem ist in einer Stadt wie Göteborg mit knapp 600.000 Einwohnern nicht so stark wie in einer Großstadt wie Stockholm, wo das Verhältnis eher bei 3/4 liegt (Eliasson 2009). Da bei diesen Berechnungen die Wohlfahrtsverluste bei der Verausgabung der Mauteinnahmen vernachlässigt werden, ist ein kleines Wohlfahrts-Maut-Verhältnis kritisch zu sehen. So müsste die Effizienz der Mautverausgabung in Göteborg mindestens 74 Prozent betragen, damit der Wohlfahrtsgewinn positiv bleibt. Nach West und Börjesson (2020) erreicht das finanzierte Hauptprojekt, ein Eisenbahntunnel, nur ein NKV von 0.45 . 


\section{Politikoptionen}

Die praktischen Erfahrungen zeigen, dass man mit der Maut gewisse Ziele erreichen kann, sie jedoch kein Allheilmittel ist. Das dem Ideal am nächsten kommende Modell in Singapur musste durch eine zusätzliche Pkw-Obergrenze erweitert werden (Tan und Wei 2020). Aufgrund der Bevölkerungsdichte und der Mittelverwendung ist der Erfolg in Stockholm ein anderer als der in Göteborg.

Widerstände sind zum einen, dass die Pkw-Nutzer die Vorteile einer Maut (reduzierte Fahrzeiten) nicht sehen, insbesondere wenn die Maut als eine Gebühr für einen Stau und nicht als Gebühr, die fließenden Verkehr ermöglicht, kommuniziert wird. Außerdem fürchten die Pkw-Nutzer (teilweise zu Recht), dass sie trotz Staureduktion verlieren. Auch die indirekt regressive Wirkung der Maut hemmt ihre Einführung.

Hohe Erhebungskosten führen dazu, dass sich die positiven Wohlfahrtseffekte der Maut reduzieren. So wäre nach Wegekostengutachten eine Pkw-Maut auf deutschen Fernstraßen in der Höhe der Erhebungskosten angebracht, welches die Wohlfahrt nicht erhöhen würde. Preiswerte Technologien mit bildhafter Kennzeichenerfassung und Rechnungserstellung am Ende des Monats wie in Schweden eingesetzt, können aus Datenschutzgründen kritisch gesehen oder abgelehnt werden. Technologien mit besserem Datenschutz sind jedoch nicht nur schwieriger zu implementieren sondern auch mit höheren Kosten verbunden. Es ist offensichtlich, dass europaweit, bundesweit, und städtevergleichend unterschiedliche Technologien (Apps, On board units) zu vermeiden sind. Der einheitliche Europäische Elektronische Mautdienst (EEMD bzw. englisch EETS) ist seit seiner Formulierung im Jahr 2011 einer Realisierung jedoch nicht näher gekommen.

Eliasson (2014) beschreibt Voraussetzungen, die erfüllt sein sollten, damit eine Maut zu einem Erfolg führt: Das Ziel einer Maut sollte, mit Unterstützung von Experten vorbereitet aber durch die Politik vorgegeben werden. Es muss operationabel sein wie beispielsweise 20 Prozent weniger motorisierter Individualverkehr in der Stadt oder oder Maximierung der Wohlfahrt durch eine Staureduktion mittels Gebühren. Da die Verkehrssysteme komplex sind, empfiehlt Eliasson (2014) ein Verkehrsmodell von Experten entwickeln zu lassen um so die Höhe und Auswirkungen der Gebühren simulieren zu können. Trotz der politischen Festlegung bei Einführung der Maut muss das System kleine Änderungen flexibel ermöglichen, um beispielsweise unerwartet auftretende Schleichverkehre schnell abstellen zu können.

Die ganze Diskussion zeigt, dass der Pfad zur Maut mit einigen Fallstricken versehen ist und nicht immer klar ist, dass das Ergebnis mit Maut besser ist als ohne. Dennoch gibt es zwei aktuelle Gründe, die die Abwägung beeinflussen sollten.

Ein Großteil der Finanzierung der Fernstraßeninfrastruktur erfolgt über die (in der Regel im Haushaltsgesetz erweiterte) Zweckbindung nach Paragraph 1 der Straßenbaufinanzierungsgesetzes durch fast die Hälfte der Einnahmen der Mineralölsteuer des Straßenverkehrs. Durch die bereits umgesetzten bzw. angekündigten staatlichen (EU-Flottenverbrauchsrichtlinie, Förderung Elektromobilität) und privaten (Volkswagen AG) Maßnahmen ist davon auszugehen, dass der Anteil der durch Elektrofahrzeuge zurückgelegten Wege auf deutschen Fernstraßen zulasten der durch konventionell (Benzin/Diesel) betriebenen Fahrzeuge stark steigen wird. Als Folge 
werden die Steuereinnahmen der Mineralölsteuer in den nächsten Jahren massiv zurückgehen. Diese Finanzierungslücke kann durch eine Fernstraßenmaut für Pkw, gegeben kostengünstigere Erhebungstechnologien als heute, geschlossen werden. Darüber hinaus erfordert halbautonomes oder autonomes Fahren eine gute Verkehrsinfrastruktur, so dass davon auszugehen ist, dass die vorhandenen Straßen (mit zusätzlichen Kosten) instandgesetzt, ausgerüstet und gewartet werden müssen.

Autonomes Fahren in der Stadt erscheint beispielsweise als Valet Parking ${ }^{5}$ in der nahen Zukunft möglich. Eine solche Anwendung jedoch verändert den Verkehr in der Stadt fundamental (Millard-Ball 2019). Überflüssige Fahrten werden schon heute vermieden, da der Fahrer Opportunitätskosten der Zeit hat. Deswegen werden die ungenutzten Pkw geparkt. Autonome Fahrzeuge könnten jedoch als Alternative zum Parken auch fahren, ohne dass dem Pkw-Nutzer Opportunitätskosten der Zeit entstehen. Sind die Fahrtkosten kleiner als die Parkkosten, wäre das auch die ökonomischere Alternative. Der Pendler mietet also keinen Parkplatz in der City, sondern schickt sein autonomes Fahrzeug morgens an den Stadtrand zum nächstgelegenen kostenlosen Parkplatz. Ist der Arbeitstag beendet, wird der Pkw wieder gerufen und startet den Weg zum Arbeitsplatz erneut. Eine solche Strategie würde die Pendelwege verdoppeln. Auch könnten autonome vernetzte Fahrzeuge Strategien entwickeln, um durch künstliche Staus entfernungsabhängige Kosten zu vermeiden. Autonome Fahrzeuge führen ohne Änderung des regulatorischen Rahmens zum Verkehrskollaps. Eine differenzierte Maut könnte Teil dieser neuen Regulierung sein.

Als Ergebnis lässt sich feststellen: Die Fernstraßenmaut für Pkw wird in absehbarer Zeit benötigt, um die Einnahmeausfälle der Mineralölsteuer zu kompensieren. Die Maut könnte zeitlich differenziert nach erwarteter Belastung werden. Oft wird eine Mautdifferenzierung nach tatsächlicher Belastung (Cramton et al. 2018; Beheshtian et al. 2020) vorgeschlagen. Hohe Transaktionskosten, stochastische Kapazitäten und Risikoaversion der Verkehrsteilnehmer sind dabei Aspekte, die beachtet werden müssen, wenn man eine solche Mautdifferenzierung mit einer, wie in Singapur praktizierten, Mautdifferenzierung nach erwarteter Belastung, vergleicht. Der wohlfahrtstheoretisch Vergleich beider Ansätze liegt bisher noch nicht vor und ist eine Forschungslücke. Mögliche und gewünschte kurzfristige Verhaltensänderungen werden schon heute im hohen Maße durch Navigationsapps erreicht. Würde man zufällige Staus auch noch mit einer hohen Maut verbinden, würden die Pkw-Fahrer im Stau doppelt negativ getroffen. Sie verlieren (unerwartet) Zeit und müssen eine (unerwartet) hohe Maut bezahlen. Dieses ist der Akzeptanz der Maut abträglich. Um die Akzeptanz zu steigern sollte man umgekehrt agieren: Die Mauthöhe sorgt für einen erwartet fließenden Verkehr. Das Zusammenbruchsrisiko durch Verkehrsunfälle oder Phantomstaus wird von allen gemeinsam versichert, indem die Maut im Falle des (unerwarteten) Verkehrszusammenbruchs gesenkt oder teilweise erstattet wird. Die Kommunikation der Maut würde positiv möglich sein: Die Maut sorgt für fließenden Verkehr, im Stau (nach umgangssprachlicher Definition, d. h. im Hyperstau nach ökonomischer Definition) hat die Maut nicht funktioniert und man bekommt sein Geld zurück. Sollte auf einzelnen Teilabschnitten das Verhältnis von Kapazität

\footnotetext{
${ }_{5}^{5}$ Fahrzeug fährt vom Übergabepunkt selbständig zum Parkplatz und kommt zur gewünschten Zeit wieder zum Übergabepunkt zurück.
} 
und Nachfrage so sein, dass ein Verkehrszusammenbruch zu gewissen Zeiten nicht vermieden werden kann, sollte dieses kommuniziert werden (Kapazität für sinnvolle Maut zu klein), die Mauthöhe nach oben begrenzt (ggf. unterhalb der Stauexternalität) und die Kompensation im Gegenzug nicht gezahlt werden. Damit dieses sinnvoll ist, muss eine kostengünstige aber den Datenschutzanforderungen entsprechende Technologie identifiziert werden. Die Technologie sollte Deutschlandweit einsetzbar und EU-weit skalierbar sein (EEMD). Städte, die eine City-Maut einführen wollen, sollte ein einfaches opt-in ermöglicht werden. So kann verhindert werden, dass jede Stadt ein eigenen System entwickeln muss. Eine einheitliche Technologie heißt jedoch nicht, dass auch die Abrechnung und Kontrolle einheitlich sein muss, hier könnte ein Wettbewerb unterschiedlicher Betreiber vorteilhaft sein. Die Erfahrungen mit Toll Collect jedoch zeigen, dass die Vor- und Nachteile des Wettbewerbs identifiziert und abgewogen werden müssen, bevor man sich für ein wettbewerbliches System oder eine Öffentlich-Private Partnerschaft (ÖPP) entscheidet. Gerade die geringe Anzahl der zu erwarteten Anbieter, und die zu Beginn geringe Anzahl der sich für eine City-Maut entscheidenden Städte, könnten selbst für einen Ausschreibungswettbewerb ungenügend und für ein ÖPP mit zu geringen Skalenerträgen und damit zu hohen Transaktionskosten verbunden sein.

Die City-Maut verfolgt keine straßendifferenzierte Feinsteuerung des Verkehrs, sondern eine Absenkung des Verkehrs in der Stadt, um ineffiziente Hyperstaus zu verhindern. Hier sollte ein Rechtsrahmen geschaffen werden, der es ermöglicht, City-Mauten einzuführen, gleichzeitig aber sowohl Spielraum für Kooperationen von Städten und Landkreisen ermöglicht, um sowohl das Gefangenendilemma der Konkurrenz sowie das Problem der politisch in den Städten nicht vertretenden Einpendler adressiert. Ziel der City-Maut ist es, den motorisierten Individualverkehr zu reduzieren, was hauptsächlich durch einen Wechsel von Pkw-Pendlern und innerstädtischen Pkw-Fahrten zum ÖPNV gelingen kann. Dieses erfordert in den meisten Städten zusätzliche Kapazitäten in den Hauptverkehrszeiten und engere Takte in den Nebenzeiten, die mit oder vor der Einführung der City-Maut angeboten werden sollten.

Funding Open Access funding provided by Projekt DEAL.

Open Access Dieser Artikel wird unter der Creative Commons Namensnennung 4.0 International Lizenz veröffentlicht, welche die Nutzung, Vervielfältigung, Bearbeitung, Verbreitung und Wiedergabe in jeglichem Medium und Format erlaubt, sofern Sie den/die ursprünglichen Autor(en) und die Quelle ordnungsgemäß nennen, einen Link zur Creative Commons Lizenz beifügen und angeben, ob Änderungen vorgenommen wurden.

Die in diesem Artikel enthaltenen Bilder und sonstiges Drittmaterial unterliegen ebenfalls der genannten Creative Commons Lizenz, sofern sich aus der Abbildungslegende nichts anderes ergibt. Sofern das betreffende Material nicht unter der genannten Creative Commons Lizenz steht und die betreffende Handlung nicht nach gesetzlichen Vorschriften erlaubt ist, ist für die oben aufgeführten Weiterverwendungen des Materials die Einwilligung des jeweiligen Rechteinhabers einzuholen.

Weitere Details zur Lizenz entnehmen Sie bitte der Lizenzinformation auf http://creativecommons.org/ licenses/by/4.0/deed.de. 


\section{Literatur}

Arnott, R. (2013). A bathtub model of downtown traffic congestion. Journal of Urban Economics, 76(1), 110-121.

Arnott, R., Kokoza, A., \& Naji, M. (2016). Equilibrium traffic dynamics in a bathtub model: a special case. Economics of Transportation, 7-8, 38-52.

Arnott, R., de Palma, A., \& Lindsey, R. (1993). A structural model of peak-period congestion: a traffic bottleneck with elastic demand. American Economic Review, 83(1), 161-179.

Beheshtian, A., Geddes, R. R., Rouhani, O., Kockelman, K., Ockenfels, A., Cramton, P., \& Do, W. (2020). Bringing the efficiency of electricity market mechanisms to multimodal mobility across congested transportation systems. Transportation Research Part A: Policy and Practice, 131, 58-69. https://doi. org/10.1016/j.tra.2019.09.021.

Brilon, W., \& Geistefeldt, J. (2010). Überprüfung der Bemessungswerte der HBS für Autobahnabschnitte außerhalb der Knotenpunkte. Forschung Straßenbau und Straßenverkehrstechnik.

Brilon, W., Geistefeldt, J., \& Regler, M. (2005). Reliability of freeway traffic flow: a stochastic concept of capacity. Prooceedings of the 16th international symposium on transportation and traffic theory. (S. 125-144).

Brilon, W., Geistefeldt, J., \& Zurlinden, H. (2007). Implementing the concept of reliability for highway capacity analysis. Transportation Research Record, 2027, 1-8.

Casady, C. B., Gómez-Ibáñez, J. A., \& Schwimmer, E. (2020). Toll-managed lanes: a simplified benefitcost analysis of seven US projects. Transport Policy, 89, 38-53. https://doi.org/10.1016/j.tranpol. 2020.02.001.

Cramton, P., Geddes, R.R., \& Ockenfels, A. (2018). Set road charges in real time to ease traffic. Nature, $560,23-25$.

Daganzo, C.F. (2007). Urban gridlock: macroscopic modeling and mitigation approaches. Transportation Research Part B: Methodological, 41(1), 49-62.

Daganzo, C.F., Gayah, V.V., \& Gonzales, E.J. (2011). Macroscopic relations of urban traffic variables: bifurcations, multivaluedness and instability. Transportation Research Part B: Methodological, 45(1), $278-288$.

Eliasson, J. (2009). A cost-benefit analysis of the stockholm congestion charging system. Transportation Research Part A: Policy and Practice, 43(4), 468-480. https://doi.org/10.1016/j.tra.2008.11.014.

Eliasson, J. (2014). The stockholm congestion charges: an overview. Working papers in transport economics 2014. (S. 7). Stockholm: CTS - Centre for Transport Studies.

Evans, A. W. (1992). Road congestion pricing: when is it a good policy? Journal of Transport Economics and Policy, 26(3), 213-243.

Fosgerau, M. (2015). Congestion in the bathtub. Economics of Transportation, 4(4), 241-255. https://doi. org/10.1016/j.ecotra.2015.08.001.

Fosgerau, M., \& Small, K. A. (2013). Hypercongestion in downtown metropolis. Journal of Urban Economics, 76(1), 122-134. https://doi.org/10.1016/j.jue.2012.12.004.

Geroliminis, N., \& Daganzo, C.F. (2008). Existence of urban-scale macroscopic fundamental digrams: some experimental findings. Transportation Research Part B: Methodological, 42(9), 759-770.

Goldmann, K., \& Sieg, G. (2020a). Economic implications of phantom traffic jams: evidence from traffic experiments. Transportation Letters. https://doi.org/10.1080/19427867.2019.1611077.

Goldmann, K., \& Sieg, G. (2020b). Quantifying the phantom jam externality: the case of an Autobahn section in germany. Münster: Institute of Transport Economics Working Paper No. 30.

Goldmann, K., \& Sieg, G. (2021). Dynamic congestion pricing and user heterogeneity. In R. Vickerman (Hrsg.), Ecyclopedia of transportation. Amsterdam: Elsevier.

Hall, F. L., \& Agyemang-Duah, K. (1991). Freeway capacity drop and the definition of capacity. Transportation Research Record, 1320, 91-98.

Millard-Ball, A. (2019). The autonomous vehicle parking problem. Transport Policy, 75, 99-108. https:// doi.org/10.1016/j.tranpol.2019.01.003.

Nakayama, A., Fukui, M., Kikuchi, M., Hasebe, K., Nishinari, K., Sugiyama, Y., Tadaki, S., \& Yukawa, S. (2009). Metastability in the formation of an experimental traffic jam. New Journal of Physics, 11, 83025 .

Puls, T. (2015). Mehr Investitionen zur Finanzierung der Bundesfernstraßen nötig. Wirtschaftsdienst, 95(6), 436-438. https://doi.org/10.1007/s10273-015-1844-5. 
Sugiyama, Y., Fukui, M., Kikuchi, M., Hasebe, K., Nakayama, A., Nishinari, K., Tadaki, S. I., \& Yukawa, S. (2008). Traffic jams without bottlenecks - experimental evidence for the physical mechanism of the formation of a jam. New Journal of Physics, 10, 33001.

Tadaki, S., Kikuchi, M., Fukui, M., Nakayama, A., Nishinari, K., Shibata, A., Sugiyama, Y., Yosida, T., \& Yukawa, S. (2013). Phase transition in traffic jam experiments on a circuit. New Journal of Physics, 15, 103034.

Tan, L., \& Wei, L. (2020). Evaluating car license auction mechanisms: theory and experimental evidence. China Economic Review, 60, 101387. https://doi.org/10.1016/j.chieco.2019.101387.

Treiber, M., \& Kesting, A. (2013). Traffic flow dynamics. Berlin, Heidelberg: Springer.

Verhoef, E. T. (2005). Speed-flow relations and cost functions for congested traffic theory and empirical analysis. Transportation Research Part A, 39, 792-812.

Vickrey, W. (2019). Types of congestion pricing models. Economics of Transportation, 20, 100140. https:// doi.org/10.1016/j.ecotra.2019.100140.

Vickrey, W.S. (1963). Pricing in urban and suburban transport. The American Economic Review, 53(2), $452-465$.

Vickrey, W.S. (1969). Congestion theory and transport investment. American Economic Review, 59, 251-261.

West, J., \& Börjesson, M. (2020). The gothenburg congestion charges: cost-benefit analysis and distribution effects. Transportation, 47(1), 145-174. https://doi.org/10.1007/s11116-017-9853-4.

Wissenschaftlicher Dienst des Bundestages (2013). Regelungskompetenz der Kommunen für die Einführung einer City-maut. https://www.bundestag.de/resource/blob/421430/df8b8d96571fa4077fbf4d979 46331eb/wd-3-140-13-pdf-data.pdf. Zugegriffen: 01.01.2020.

Yuan, K., Knoop, V.L., Leclercq, L., \& Hoogendoorn, S.P. (2017). Capacity drop: a comparison between stop-and-go wave and standing queue at lane-drop bottleneck. Transportmetrica B: Transport Dynamics, 5(2), 145-158. 